\title{
Effect of Astragalus membranaceus extract on diabetic nephropathy
}

\author{
Jiman Kim, Eulsun Moon ${ }^{1}$ and Seungwon Kwon ${ }^{2}$ \\ Kyunghee-saeng Korean Medicine Clinic, Seoul, Korea ${ }^{1}$ Seoul 365 Medical Clinic, Seoul, Korea ${ }^{2}$ Department of \\ Cardiovascular and Neurologic Diseases, College of Korean Medicine, KyungHee University, 1 Hoegi-dong, \\ Dongdaemun-gu, Seoul 130-702, Korea
}

Correspondence

should be addressed

to S Kwon

Email

kkokkottung@hanmail.net

\section{Summary}

Diabetic nephropathy, a microvascular complication of diabetes, is a progressive kidney disease caused by angiopathy of the capillaries in the kidney glomeruli. Herein, we report a case of a 62-year-old patient with a 30 year history of diabetes, who showed a substantial improvement in diabetic nephropathy on administration of $30 \mathrm{~g}$ of Astragalus membranaceus extract per day. After 1 month, estimated glomerular filtration rate increased from 47 to $72 \mathrm{ml} / \mathrm{min}$ per $1.73 \mathrm{~m}^{2}$ and was subsequently maintained at the 1-month follow-up. Urinary protein levels also decreased following treatment. Herein, we present and discuss the evidence and mechanism of $A$. membranaceus on diabetic nephropathy in this patient.

\section{Learning points:}

- Diabetic nephropathy is a progressive kidney disease.

- Angiotensin-converting enzyme (ACE) inhibitors or angiotensin receptor blockers (ARBs) are currently used to prevent and delay the progression of diabetic nephropathy. However, their effects are not sufficient to prevent a decline in kidney function.

- Furthermore, combination therapy with an ACE inhibitor and an ARB can produce adverse effects without additional benefits.

- In the early phase of diabetic nephropathy, administration of Astragalus membranaceus can be a therapeutic option.

\section{Background}

Diabetic nephropathy, a microvascular complication of diabetes, is a progressive kidney disease caused by angiopathy of the capillaries in the kidney glomeruli. The principal cause of diabetic nephropathy, a prime indicator for dialysis, is long-standing hypertensive diabetes mellitus, characterized pathophysiologically by glomerular hyperfiltration. Angiotensin-converting enzyme (ACE) inhibitors and angiotensin receptor blockers (ARBs), both commonly used in preventing diabetic nephropathy, act on this pathophysiology (1). However, the preventative and therapeutic effect of combined ACE inhibition and ARB therapy on diabetic nephropathy is still insufficient to maintain kidney function (2). In addition, the combined use of ACE inhibitors with ARBs offers no discernible benefit and is associated with an increase in adverse effects (3). In this case study, we present a patient with established diabetes showing a dramatic decrease in proteinuria and increase in estimated glomerular filtration rate (eGFR) with substantial improvement in diabetic nephropathy on administration of Astragalus membranaceus extract. Furthermore, we discuss the evidence and mechanism of effect of A. membranaceus on the pathophysiology of diabetic nephropathy.
(C) 2014 The authors http://www.edmcasereports.com Published by Bioscientifica Ltd 


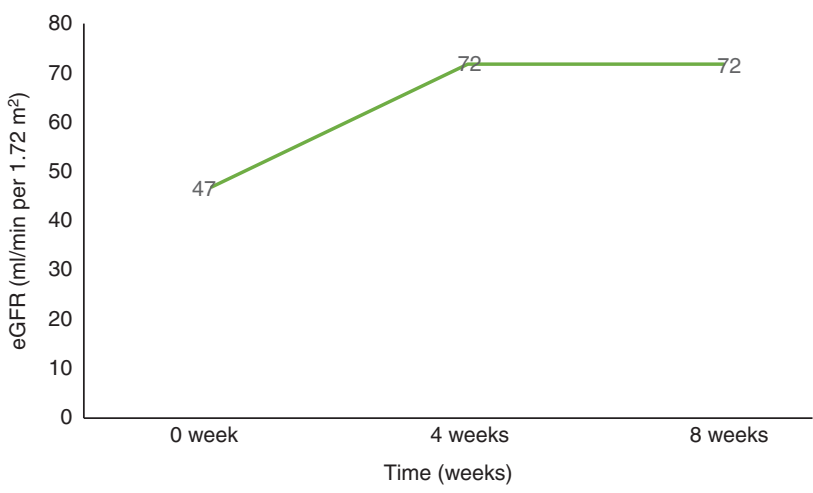

Figure 1

Changes in estimated glomerular filtration rate (eGFR). 0 week: April 7, 2014; 4 weeks: May 3, 2014; 8 weeks: June 7, 2014.

\section{Case presentation}

A 62-year-old man (weight, $68 \mathrm{~kg}$; height, $170 \mathrm{~cm}$; waist, $86.36 \mathrm{~cm}$ ) with controlled diabetes mellitus, hypertension, dyslipidemia, diabetic retinopathy, diabetic foot (foot ulcer), and early diabetic nephropathy presented at Kyunghee-saeng Korean Medicine Clinic. He was previously diagnosed with type 2 diabetes at another hospital and had been receiving oral hypoglycemic agents to control blood glucose levels from 1984 until 2000 when he suffered a stroke. His blood glucose levels were subsequently found to be poorly controlled and therefore insulin injection therapy was recommended. At the same time, diabetic retinopathy was also detected, which was treated by a combination of laser photocoagulation and an adjusted calcium dobesilate dose of $250 \mathrm{mg} /$ day. The patient subsequently modified his lifestyle and continued his medication. In 2011, amputation of the right second and third toes was performed due to the presence of diabetic foot ulcers. Insulin therapy was discontinued in January 2012 because of several hypoglycemic events and oral hypoglycemic therapy was resumed (sitagliptin $100 \mathrm{mg} /$ day, metformin 500 mg/day). However, in November 2013, diabetic nephropathy corresponding to stage 3 chronic kidney disease (CKD) was detected by routine medical checkups and treatment with Perindopril commenced. This failed to improve kidney function and he presented at our clinic on April 7, 2014, seeking to recover his renal function level with herbal medicine.

\section{Investigation}

At presentation, fasting levels of glucose and $\mathrm{HbA} 1 \mathrm{c}$ were $4.884 \mathrm{mmol} / \mathrm{l}(88 \mathrm{mg} / \mathrm{dl})$ and $42 \mathrm{mmol} / \mathrm{mol}$ (6.0\%), respectively, showing adequate blood glucose control. The eGFR was $47 \mathrm{ml} / \mathrm{min}$ per $1.73 \mathrm{~m}^{2}$ as examined by the Modification of Diet in Renal Disease (MDRD) equation, indicating stage $3 \mathrm{CKD}$. This corresponded with a serum creatinine level of $122.0 \mu \mathrm{mol} / 1(1.6 \mathrm{mg} / \mathrm{dl})$. The urinary protein levels were $53 \mathrm{mg} / \mathrm{dl}$.

\section{Treatment}

The patient continued to receive their existing medications: hypoglycemic agents (sitagliptin $100 \mathrm{mg} / \mathrm{day}$, metformin $500 \mathrm{mg} /$ day), antilipidemic agent (atorvastatin $10 \mathrm{mg} /$ day), antihypertensive agents (diltiazem $180 \mathrm{mg} /$ day, perindopril $16 \mathrm{mg} /$ day), antiplatelet agents (aspirin $100 \mathrm{mg} /$ day, clopidogrel $75 \mathrm{mg} /$ day), Ginkgo biloba extract $160 \mathrm{mg} /$ day, and calcium dobesilate $750 \mathrm{mg} /$ day. In addition, the patient took $A$. membranaceus extract (30 g/day) three times a day until June 7, 2014.

\section{Outcome and follow-up}

During the administration of A. membranaceus extract, regular tests were performed once a month to check for its effect on diabetic nephropathy. After 1 month, eGFR increased from 47 to $72 \mathrm{ml} / \mathrm{min}$ per $1.73 \mathrm{~m}^{2}$, which was maintained at the 1-month follow-up (Fig. 1). After 1 month, urinary protein levels decreased from 53 to $27 \mathrm{mg} / \mathrm{dl}$ but were increased slightly to $38 \mathrm{mg} / \mathrm{dl}$ at follow-up (Fig. 2).

Other parameters such as, glucose level, HbA1c and cholesterol were within the normal range during the follow-up period.

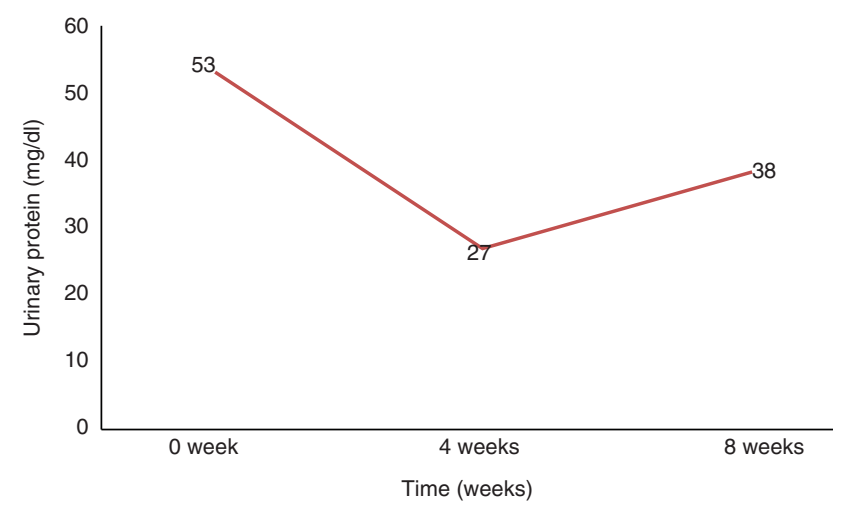

Figure 2

Changes in urinary protein. $X$ axis, time in weeks; $Y$ axis, urinary protein (mg/dl); 0 week, April 7, 2014; 4 weeks, May 3, 2014; 8 weeks, June 7, 2014. 


\section{Discussion}

We demonstrate an improvement in eGFR in a patient with established diabetic nephropathy after treatment with A. membranaceus extract for 2 months. With A. membranaceus extract administration, kidney function improved from stage $3 \mathrm{CKD}$, which is indicative of chronic renal failure, to stage 2, thus reversing renal failure. Furthermore, urinary protein output also decreased compared with initial levels.

Diagnosis of diabetic nephropathy is based on the estimation of urinary albumin or eGFR by the CockroftGault or MDRD equation. Stages of CKD as defined by eGFR are from stage 1 to 5 (stage 1: $\geq 90$; stage 2: 60-89; stage 3: 30-59; stage 4: $15-29$, and stage $5:<15 \mathrm{ml} / \mathrm{min}$ per $1.73 \mathrm{~m}^{2}$ respectively). In this case, the patient's eGFR corresponded to stage $3 \mathrm{CKD}$ which was indicative of chronic renal failure. Despite 5 months of ACE inhibitor administration, controlled blood pressure, and controlled blood glucose level, there was no improvement in disease status. Therefore, the patient gave his consent to determine the effect of herbal medication to improve his condition.

We chose A. membranaceus extract based on the following reasons. A meta-analysis (4) showed that A. membranaceus injection had a more therapeutic effect, including renal protective effect and systemic state improvement, in diabetic nephropathy patients than in control patients in the clinical situation. Another metaanalysis (5) focusing on animal models suggested that A. membranaceus is effective in reducing fasting blood glucose and albuminuria levels, reversing glomerular hyperfiltration and ameliorating the pathological changes in early diabetic nephropathy models. An experimental study (6) (7) suggested that the mechanisms of A. membranaceus action in diabetic nephropathy are a reduction in the mRNA level of nuclear factor kappa B $(N F \kappa B)$, which has been suggested to play a key role in the pathogenesis of diabetic nephropathy, and an increase in inhibitory NFKB protein mRNA expression in the renal cortex. Inhibition of high glucose-induced early mesangial cell proliferation and advanced glycation end-product (AGE)-induced endothelial cell apoptosis via effects of calycosin and calycosin-7-O- $\beta$-D-glucoside, two major isoflavonoids present in A. membranaceus, are also suggested to contribute to the beneficial effect of A. membranaceus on diabetic nephropathy (8).

Diabetic nephropathy is the single most common cause of end-stage renal disease (ESRD) (9). It is a progressive disease involving multiple factors including metabolic and hemodynamic alterations, oxidative stress, activation of the renin-angiotensin system, and inflammation (release of cell adhesion molecules, growth factors, chemokines, and pro-inflammatory cytokines) (10). In the present case, there was an improvement in diabetic nephropathy following administration of A. membranaceus for 2 months, although from this data we cannot draw conclusion of which pathway was affected.

Because of the short follow-up period, we also cannot be certain of the long-term effect of A. membranaceus administration on this patient's progressive nephropathy. However, this case provides compelling evidence to support the administration of A. membranaceus as a treatment to prevent the progression of diabetic nephropathy and improve kidney function, especially in the early stages of the disease.

\section{Declaration of interest}

The authors declare that there is no conflict of interest that could be perceived as prejudicing the impartiality of the research reported.

\section{Funding}

This research did not receive any specific grant from any funding agency in the public, commercial or not-for-profit sector.

\section{Patient consent}

Written informed consent has been obtained from the patient for publication of the submitted article.

\section{Author contribution statement}

Jiman Kim treated this patient. Seungwon Kwon wrote this manuscript and reviewed this case. Eulsun Moon was involved in critical revision of the manuscript.

\section{References}

1 Wang SL, Head J, Stevens L \& Fuller JH 1996 Excess mortality and its relation to hypertension and proteinuria in diabetic patients. The World Health Organization multinational study of vascular disease in diabetes. Diabetes Care 19 305-312. (doi:10.2337/diacare.19.4.305)

2 American Diabetes Association 2013 Standards of medical care in diabetes - 2013. Diabetes Care 36 (Suppl 1) S11-S66. (doi:10.2337/dc13-S004)

3 Vivian E \& Mannebach C 2013 Therapeutic approaches to slowing the progression of diabetic nephropathy - is less best? Drugs in Context 2013 212249. (doi:10.7573/dic.212249)

4 Li M, Wang W, Xue J, Gu Y \& Lin S 2011 Meta-analysis of the clinical value of Astragalus membranaceus in diabetic nephropathy. Journal of Ethnopharmacology 133 412-419. (doi:10.1016/j.jep.2010.10.012)

5 Zhang J, Xie X, Li C \& Fu P 2009 Systematic review of the renal protective effect of Astragalus membranaceus (root) on diabetic nephropathy in animal models. Journal of Ethnopharmacology $\mathbf{1 2 6}$ 189-196. (doi:10.1016/j.jep.2009.08.046) 
6 Zhang YW, Wu CY \& Cheng JT 2007 Merit of Astragalus polysaccharide in the improvement of early diabetic nephropathy with an effect on mRNA expre-

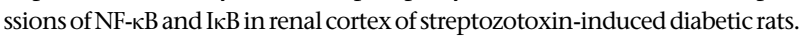
Journal of Ethnopharmacology 114 387-392. (doi:10.1016/j.jep.2007.08.024)

7 Gui D, Huang J, Guo Y, Chen J, Chen Y, Xiao W, Liu X \& Wang N 2013 Astragaloside IV ameliorates renal injury in streptozotocin-induced diabetic rats through inhibiting NF-кB-mediated inflammatory genes expression. Cytokine 61 970-977. (doi:10.1016/j.cyto.2013.01.008)

8 Tang D, He B, Zheng ZG, Wang RS, Gu F, Duan TT, Cheng HQ \& Zhu Q 2011 Inhibitory effects of two major isoflavonoids in Radix Astragali on high glucose-induced mesangial cells proliferation and AGEs-induced endothelial cells apoptosis. Planta Medica 77 729-732. (doi:10.1055/ s-0030-1250628)

9 Navarro-González JF, Jarque A, Muros M, Mora C \& García J 2009 Tumor necrosis factor- $\alpha$ as a therapeutic target for diabetic nephropathy. Cytokine \& Growth Factor Reviews 20 165-173. (doi:10.1016/ j.cytogfr.2009.02.005)

10 Duran-Salgado MB \& Rubio-Guerra AF 2014 Diabetic nephropathy and inflammation. World Journal of Diabetes 5 393-398. (doi:10.4239/ wjd.v5.i3.393)

Received in final form 6 July 2014

Accepted 12 September 2014 